\title{
Bayesian Mechanism Based Inter-Operator Base Station Sharing for Energy Saving
}

\author{
Yanan Bao*, Jian $\mathrm{Wu}^{\dagger}$, Sheng Zhou ${ }^{\dagger}$, Zhisheng Niu ${ }^{\dagger}$ \\ *Dept. of Computer Science, University of California, Davis 95616, USA \\ Email: ynbao@ucdavis.edu \\ ${ }^{\dagger}$ Dept. of Electronic Engineering, Tsinghua University, Beijing 100084, China \\ Email:wujian09@mails.tsinghua.edu.cn, \{sheng.zhou, niuzhs\}@tsinghua.edu.cn
}

\begin{abstract}
In cellular networks, the coverage of base stations (BSs) belonging to different operators often overlaps. As a result, when the traffic load is lower than the peak volume, there are opportunities to turn off a subset of BSs to save power, potentially from different operators, leaving their users to be served by other BSs. Because in this case the active BSs can be shared among different operators, it is rational to assume that operators are self-interested and hold their own private information, such as their own traffic loads. In this paper, we consider the problem of how to motivate operators to cooperate and reveal their private information such that the overall utility can be maximized, which is also called social efficiency in mechanism design. A new BS utility model that depends on the BS's energy consumption is proposed. Based on this, a game theoretic mechanism with money transfer between operators is designed, which has been proved to be incentive compatible and budget-balanced. Simulation results under various traffic load distributions show that when the operators have similar traffic load distributions, they would like to participate the cooperation voluntarily.
\end{abstract}

\section{INTRODUCTION}

The ever-increasing traffic demand in cellular networks has triggered a large expansion of network infrastructures, which results in substantial increase in energy consumption. For example, China mobile, the largest operator in China, has an electricity consumption of over 13 billion $\mathrm{KWH}$ in 2011 [1], with an electricity bill of over $\$ 1$ billion this year. Therefore, nowadays, operators have incentive to save energy from both environmental and monetary perspectives.

BS sleeping is an effective mechanism to reduce energy consumption of cellular networks. It is found that $60-80 \%$ of energy consumption in cellular networks comes from BSs [2]. Generally, a sleeping BS reduces its energy consumption by $1 / 2-2 / 3$ compared with its active mode [3]. Moreover, the capacity of a BS is designed for its peak traffic. But most of the time, the actual traffic is much lower than the peak traffic, as shown in Fig.1. Therefore, it is reasonable to let a subset of BSs go to sleep when the traffic is below a certain threshold. When one BS sleeps, its customers need to be covered and served by BSs either from the same operator or from another operator.

BS cooperation among operators has the potential to become more popular in the future because of its capability to reduce energy consumption. Nowadays, an operator typically uses roaming to cover its users in areas where its own infrastructure does not exist. On the other hand, the coverage of BSs from different operators overlaps densely, especially in highly populated urban area. Keeping all the BSs from all operators active actually wastes resource during off-peak hours. Therefore, during off-peak hours, one can save much energy by switching off a subset of BSs and transferring the load (i.e., roaming) to BSs of other operators [4]. This is our motivation to study inter-operator BS sharing.

In our work, we assume that the BSs are self-interested and we study how to motivate the operators to share their BSs and under what conditions the cooperation can form. The main contributions of this paper are as follows:

- Assuming that each operator holds its own load information and aims to maximize its own profit, a game theoretic mechanism is designed to realize BS sharing among operators, which can achieve incentive compatibility, budget balance, and participation constraints at the same time.

- Under the resource-sufficient scenario, different from the previous work where the utility is determined by immediate payment from users, a new BS utility model is considered here. In this model, the utility of a BS depends on its energy consumption which is more practical and realistic.

- The conditions under which operators can form a cooperation is analyzed and evaluated through simulation.

The remainder of this paper is organized as follows. In Section II, we introduce the related work. Section III presents the energy consumption model, traffic model and utility model for BSs. Section IV introduces the Bayesian mechanism we designed to solve the BS sharing problem. Simulations and performance evaluation are given in Section V. Finally, Section VI concludes the paper and discusses the future work.

\section{RELATED WORK}

BS sleeping is an effective way to realize energy saving during the low-traffic hours [2], [5], [6]. If a BS goes to sleep, the neighboring $\mathrm{BSs}$ or the $\mathrm{BSs}$ from different hierarchical levels can be used to help the sleeping BS. However, there is only limited work on the possibility of BS sharing among operators. It is shown by [4] that $29 \%$ of the operation energy cost can be saved though BS sharing among operators. The authors also mention that it will be an interesting problem to study under what conditions the self-interested operators 


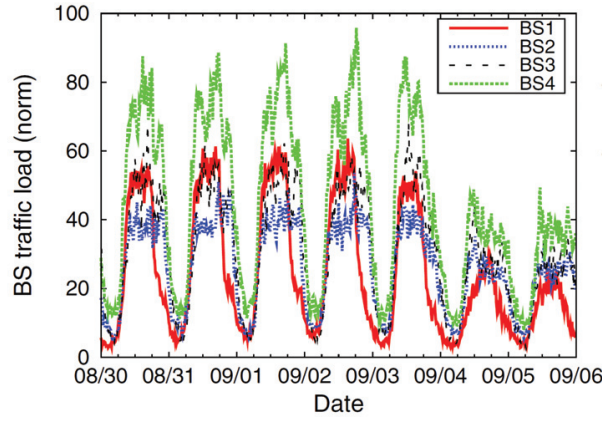

Fig. 1. One-week traffic pattern from 4 BSs in 4 regions [14].

would like to cooperate with others from a game theoretic perspective.

To answer this question, based on the BS sleeping model, the authors analyze when self-interested operators would agree to share their resources and serve the customers of others [7]. In [8], the authors investigate the spectrum sharing with other operators without BS sleeping.

In our work, rather than studying the conditions to establish cooperation, we design a game theoretic mechanism that implements cooperation. This allows us to implement the socially efficient outcome despite the fact that BSs only have private information about their loads. The most common mechanisms are Vickrey-Clark-Groves mechanisms [9]-[11]. Yet, they do not necessarily achieve budget balance. Instead, we use Bayesian mechanism design, in particular the expected externality mechanism introduced in [12], [13]. This mechanism allows us to achieve the socially efficient outcome, incentive compatibility, budget balance and sometimes participation constraints.

\section{BASE STATION MOdel}

We consider $N$ BSs in total and each of them belongs to a different operator. We start from the energy consumption model, and then go to the traffic model and the utility model.

\section{A. Energy Consumption Model}

In our simplified model, the power consumption of a BS is determined only by the realtime traffic load. Denote the traffic load of BS $i$ by $l_{i}$. We have the power consumption of BS $i$ as a function of $l_{i}$ as follows [3]:

$$
P_{i}^{c}\left(l_{i}\right)= \begin{cases}P_{i}^{o}+C_{i}^{p} l_{i}, & \text { for } 0<l_{i} \leqslant l_{i}^{\max } \\ P_{i}^{\text {sleep }}, & \text { for } l_{i}=0,\end{cases}
$$

where $P_{i}^{\text {sleep }}$ is the power consumption in sleeping mode, $P_{i}^{o}$ is the static power consumption in active mode and $C_{i}^{p} l_{i}$ is the power consumption related to load for BS $i$. For the ease of analysis, we do not consider the BS switching cost in this work.

\section{B. Traffic Model}

In this preliminary work, time is divided into slots and each slot has a duration of $d$. Without BS sharing, the load generated in the current slot will be served in the following slot. We assume that at each time slot the traffic load is i.i.d. with a certain distribution. Therefore, the BS cooperation problem can be divided into temporally uncorrelated games. Results based on this simplified model provide some intuitions and it is also applicable to the general cases. This assumption can also be explained by the following reasons: Even though from a large temporal granularity, the traffic pattern is smooth and predictable, the small scale traffic variation is random and unpredictable. Moreover, compared with the large cells, the traffic varies dramatically in small cells (, e.g. femtocells) which will be widely deployed with the upgrade of cellular networks.

Another assumption about the traffic is that it can be transferred between operators at the end of the current time slot with a small cost. In a cloud-based architecture, virtual resource makes load transfer between BSs much easier [15]. After load transfer, a subset of BSs may have no load to serve and a subset of BSs may have more load to serve in the following slot. Each BS would like to serve the load transferred from the other BSs, if it is paid and has sufficient capability.

Traffic loads are the private information for BSs. A BS knows its own realtime traffic load at the end of the current slot, but it can never infer or predict the other BSs' traffic loads.

\section{Utility Model}

We consider the scenario where the networks are not resource-limited, but demand-limited. This is common during the off-peak hours in urban areas and even during the whole day in some rural areas, due to the requirement enforced to network deployment. In this case, a BS has the ability to meet all the requirements of its consumers and doesn't need to consider the QoS (Quality of Service). Therefore, all the requests from users will be served, and the utility of an operator is determined by the payment from users, which is independent of operators' actions, and the resource expenditure for running its BSs. Most of previous works assume a positive immediate utility if BSs serve a user and a penalty otherwise, which is neither practical nor realistic in the resource sufficient networks.

Denote the fee for using electricity by $\Delta_{e}$ per Joule. Considering a generic time slot, when there is load transfer at the end of the slot, both the sender and the receiver have a cost of $\Delta_{t}$ per unit of load. The cost can be caused by the additional energy consumption for load transferring. The payment BS $i$ receives from other BSs is denoted by $t_{i}$. There are two cases:

- BS $i$ transfers its load $l_{i}$ to other BSs. In the following time slot, it can go to sleep and its utility is

$$
\begin{aligned}
U_{i}\left(l_{i}\right) & =-\Delta_{e} P_{i}^{c}(0) d-\Delta_{t} l_{i}+t_{i} \\
& =-\Delta_{e} P_{i}^{\text {sleep }} d-\Delta_{t} l_{i}+t_{i}
\end{aligned}
$$

- BS $i$ is active and accepts load from other BSs. Denote 
the load BS $i$ receives from other BSs by $l_{i}^{t}$. When $l_{i}^{t}<0$, BS $i$ transfers its own load to the other BSs.

In the following time slot, the utility of BS $i$ is

$$
U_{i}\left(l_{i}, l_{i}^{t}\right)=-\Delta_{e} P_{i}^{c}\left(l_{i}+l_{i}^{t}\right) d-\Delta_{t} l_{i}^{t}+t_{i},
$$

where

$$
0<l_{i}+l_{i}^{t} \leqslant l_{i}^{\max } .
$$

\section{BAYESIAN MEChanism Design}

The private information of each BS is its own traffic load. This private information varies over time. Since we assume that loads are temporally independent, this generates an i.i.d. sequence of Bayesian games. The problem is how to select a subset of BSs to be active, how to transfer the load in each slot between BSs, and how to decide the payment $t_{i}$-s to meet the constraints of the mechanism.

The designed mechanism works as follows. At the end of each time slot, all BSs announce their own loads and a subset of the BSs are selected by either a coordinator or the BSs to be active and the others to be sleeping. The sleeping BSs will transfer their announced loads (which may not be the true loads if some BSs don't tell the truth) to the active BSs. The active BSs will get paid according to the announced loads of all BSs.

The announced load of BS $i$ is denoted by $\hat{l}_{i}$, and $\hat{l}_{i}$ is chosen to maximize its utility by BS $i$. Considering $N$ BSs, we have $\hat{L}=\left[\hat{l}_{1}, \hat{l}_{2}, \ldots, \hat{l}_{N}\right]$. Note that the real loads are denoted by $L=\left[l_{1}, l_{2}, \ldots, l_{N}\right]$, and the transferred loads are denoted by $L^{t}=\left[l_{1}^{t}, l_{2}^{t}, \ldots, l_{N}^{t}\right]$.

For BS $i$, a boolean variable $y_{i}$ is used to indicate whether it is chosen to be active or not. If BS $i$ is active, $y_{i}=1$; otherwise, $y_{i}=0$. For the ease of notation, $Y=\left[y_{1}, y_{2}, \ldots, y_{N}\right]$. The received payment for all the BSs are denoted by $T$, i.e., $T=\left[t_{1}, t_{2}, \ldots, t_{N}\right]$.

Given a set of BSs and their announced loads $\hat{L}$, the social choice function $x(\hat{L})$ gives the output $\left(Y, L^{t}, T\right)$. The function $x(\bullet)$ includes all the parameters of different BSs in the set. Considering the payment $t_{i}(\hat{L})$ as a function of the announced load $\hat{L}$, the utility of BS $i$ is

$$
u_{i}\left(x(\hat{L}), l_{i}\right)=-\Delta_{e} P_{i}^{c}\left(l_{i}+l_{i}^{t}\right) d-\Delta_{t}\left|l_{i}^{t}\right|+t_{i}(\hat{L}) .
$$

\section{A. Mechanism Constraints}

An applicable mechanism should satisfy the following requirements: socially efficient, incentive for telling the true loads, budget-balanced and participation constraints.

1) Social efficiency: The overall utility covering all the operators is maximized. Usually the BSs with the highest loads and the highest energy efficiency are more likely to be chosen as active BSs.

2) Incentive compatibility: Revealing their true traffic loads is a Bayesian Nash equilibrium of the mechanism.

3) Budget balance: The inter-payments between operators sum up to 0 , i.e.,

$$
\sum_{i \in N} t_{i}(\hat{L})=0
$$

This means there is no deficit or surplus in each time slot.

4) Participation constraints: Operators would like to join this mechanism voluntarily.

For a mechanism, three kinds of participation constraints exist: ex post, interim and ex ante. The ex post participation constraint means that even though the load information is known by all the operators and there is no private information, they still would like to join the game and share their BSs. This constraint can be written as

$$
u_{i}\left(x(L), l_{i}\right) \geqslant-\Delta_{e} P_{i}^{c}\left(l_{i}\right), \quad \forall i \in 1, \ldots, N .
$$

The interim participation constraint means when a BS only knows its own load, it would like to join the game to get a higher expected utility based on the distribution of others' loads. Since BS $i$ only knows its own load, the loads of other BSs are $N-1$ random variables to this BS. Denote the $N-1$ random variables by $\bar{L}_{-i}$, where

$$
\bar{L}_{-i}=\left[\bar{l}_{1}, \bar{l}_{2}, \ldots, \bar{l}_{i-1}, \bar{l}_{i+1}, \ldots, \bar{l}_{N}\right] .
$$

The interim participation constraint requires

$$
\mathrm{E}_{\bar{L}_{-i}}\left[u_{i}\left(x(L), l_{i}\right)\right] \geqslant-\Delta_{e} P_{i}^{c}\left(l_{i}\right), \quad \forall i \in 1, \ldots, N .
$$

The ex ante participation constraint means when a BS does not know its own load as well as others' loads, it would like to join the game to get a higher expected utility based on the distribution of all the loads , i.e.,

$$
\mathrm{E}_{L}\left[u_{i}\left(x(L), l_{i}\right)\right] \geqslant \mathrm{E}_{l_{i}}\left[-\Delta_{e} P_{i}^{c}\left(l_{i}\right)\right], \forall i \in 1, \ldots, N .(12)
$$

\section{B. Bayesian Mechanism Solutions}

1) Socially Efficient Choice: The computation of $x(\hat{L})$ involves an integer programming problem as follows.

$$
\begin{aligned}
& \underset{\left(Y, L_{t}\right)}{\operatorname{argmin}} \sum_{i}^{N} \Delta_{e}\left[y_{i}\left(P_{i}^{o}+C_{i}^{p}\left(\hat{l}_{i}+l_{i}^{t}\right)\right)+\left(1-y_{i}\right) P_{i}^{\text {sleep }}\right] d+\Delta_{t}\left|l_{i}^{t}\right| \\
& \text { s.t. } \quad 0 \leqslant \hat{l}_{i}+l_{i}^{t} \leqslant l_{i}^{\text {max }} ; \\
&\left(1-y_{i}\right)\left(\hat{l}_{i}+l_{i}^{t}\right)=0 ; \\
& y_{i} \in\{0,1\}, \quad \forall i \in 1, \ldots, N .
\end{aligned}
$$

This is a NP-hard problem. However, since usually there are only 3-5 operators in a certain region, the problem is computable in our case.

In a special case when the parameters $\left(P_{i}^{o}, C_{i}^{p}, P_{i}^{\text {sleep }}\right)$ are the same for all BSs, it is the well-known bin packing problem [16]. Moreover, if $\sum_{j}^{N} \hat{l}_{j} \leqslant l_{i}^{\max }, \forall i \in 1, \ldots, N$, which means all the BSs are lightly loaded, the most heavily loaded BS is chosen to be active, i.e.

$$
I_{a}=\arg \max _{i} \hat{l}_{i} \text { for } i=1,2, \ldots, N .
$$

The optimal solution is

$$
y_{i}^{*}=\left\{\begin{array}{cc}
0, & \text { if } i \neq I_{a} \\
1, & \text { if } i=I_{a} .
\end{array}\right.
$$


and

$$
l_{i}^{t^{*}}= \begin{cases}-\hat{l}_{i}, & \text { if } i \neq I_{a} ; \\ \sum_{j \neq I_{a}} \hat{l}_{j}, & \text { if } i=I_{a} .\end{cases}
$$

2) Payment Calculation: Before being paid, BS $i$ 's utility is

$$
v_{i}\left(x(\hat{L}), l_{i}\right)=-\Delta_{e} P_{i}^{c}\left(l_{i}+l_{i}^{t}\right) d-\Delta_{t}\left|l_{i}^{t}\right| .
$$

By setting the payment as

$$
\begin{aligned}
t_{i}(\hat{L})= & \mathrm{E}_{\bar{L}_{-i}}\left[\sum_{j \neq i} v_{j}\left(x\left(\hat{l}_{i}, \bar{L}_{-i}\right), \bar{l}_{j}\right)\right] \\
& -\frac{1}{N-1} \sum_{k \neq i} \mathrm{E}_{\bar{L}_{-k}}\left[\sum_{j \neq k} v_{j}\left(x\left(\hat{l}_{k}, \bar{L}_{-k}\right), \bar{l}_{j}\right)\right],
\end{aligned}
$$

we can get the Bayesian incentive compatible and budgetbalanced mechanism [12], [13]. For $\mathrm{E}_{\bar{L}_{-i}}[\bullet]$, the expectation is taken on the vector $\bar{L}_{-i}$. Since the loads are independent with each other,

$$
\mathrm{E}_{\bar{L}_{-i}}[\bullet]=\mathrm{E}_{\bar{l}_{1}} \mathrm{E}_{\bar{l}_{2}} \ldots \mathrm{E}_{\bar{l}_{i-1}} \mathrm{E}_{\bar{l}_{i+1}} \ldots \mathrm{E}_{\bar{l}_{N}}[\bullet] .
$$

\section{Proof of incentive compatibility and budget balance}

Since $x(\hat{L})$ is the social choice to maximize the total utility of all BSs, we have

$$
\sum_{i=1}^{N} v_{i}\left(x(\hat{L}), \hat{l}_{i}\right) \geqslant \sum_{i=1}^{N} v_{i}\left(x\left(\hat{L}^{\prime}\right), \hat{l}_{i}\right),
$$

for any $\hat{L}^{\prime}$. When all the other BSs $j \neq i$ announce their true load, we have

$$
\begin{array}{r}
\mathrm{E}_{\bar{L}_{-i}}\left[v_{i}\left(x\left(l_{i}, \bar{L}_{-i}\right), l_{i}\right)+\sum_{j \neq i} v_{j}\left(x\left(l_{i}, \bar{L}_{-i}\right), \bar{l}_{j}\right)\right] \geqslant \\
\mathrm{E}_{\bar{L}_{-i}}\left[v_{i}\left(x\left(\hat{l}_{i}^{\prime}, \bar{L}_{-i}\right), l_{i}\right)+\sum_{j \neq i} v_{j}\left(x\left(\hat{l}_{i}^{\prime}, \bar{L}_{-i}\right), \bar{l}_{j}\right)\right], \quad \forall \hat{L}^{\prime} .
\end{array}
$$

Since $\frac{1}{N-1} \sum_{k \neq i} \mathrm{E}_{\bar{L}_{-k}}\left[\sum_{j \neq k} v_{j}\left(x\left(\hat{l}_{k}, \bar{L}_{-k}\right), \bar{l}_{j}\right)\right]$ is independent of $\hat{l}_{i}, \hat{l}_{i}=l_{i}$ is the optimal announced load for BS $i$ to maximize its expected utility $\mathrm{E}_{\bar{L}_{-i}}\left[v_{i}\left(x\left(\hat{l}_{i}, \bar{L}_{-i}\right), l_{i}\right)+\right.$ $\left.t_{i}\left(\hat{l}_{i}, \bar{L}_{-i}\right)\right]$. Therefore the mechanism is incentive compatible and every BS will reveal the true load.

For notational ease, define

$$
\theta_{i}\left(\hat{l}_{i}\right)=\mathrm{E}_{\bar{L}_{-i}}\left[\sum_{j \neq i} v_{j}\left(x\left(\hat{l}_{i}, \bar{L}_{-i}\right), \bar{l}_{j}\right)\right] .
$$

We have

$$
\begin{aligned}
\sum_{i=1}^{N} t_{i}(\hat{L}) & =\sum_{i=1}^{N} \theta_{i}\left(\hat{l}_{i}\right)-\sum_{i=1}^{N} \frac{1}{N-1} \sum_{k \neq i} \theta_{i}\left(\hat{l}_{i}\right) \\
& =\sum_{i=1}^{N} \theta_{i}\left(\hat{l}_{i}\right)-\sum_{i=1}^{N} \frac{1}{N-1}(N-1) \theta_{i}\left(\hat{l}_{i}\right)=0 .
\end{aligned}
$$

Therefore the mechanism is also budget-balanced.

Myerson-Satterthwaite theorem [17] shows that in the case of two operators, it is impossible to satisfy interim participation constraint. In our case, the ex ante and interim participation constraint may or may not be satisfied as shown by simulations.

\section{Simulations and Performance evaluation}

In the simulation, a homogeneous cellular network including two BSs is considered, and the two BSs belong to two different operators. They have the same parameters as follows: $\Delta_{e}=1 / \mathrm{J}, P^{\text {sleep }}=50 \mathrm{w}, P^{o}=100 \mathrm{w}, C^{p}=10 \mathrm{~J} / \mathrm{Mb}$, $\Delta_{t}=0.1 \mathrm{~s} / \mathrm{Mb}, l^{\max }=15 \mathrm{Mb} / \mathrm{s}$. We assume the normal load is uniformly distributed in $[0,10 \mathrm{Mb} / \mathrm{s}]$. The duration of one time slot is 1 s, i.e., $d=1$ s. Next we analyze five different cases: A) both BSs have the normal load; B) one of them is lightly loaded than the normal load; C) one of them is heavily loaded than the normal load; D) both of them are heavily loaded; E) both of them are lightly loaded.

\section{A. Both BSs have normal load}

In this case, we assume that the load of both BSs is uniformly distributed over $[0,10 \mathrm{Mb} / \mathrm{s}]$.

The total utility of BS 1 including payment is plotted in Fig. 2. Given a sample of $\left(l_{1}, l_{2}\right)$, sometimes cooperating leads to utility loss, which means the ex post participation constraint is unsatisfied. When $l_{1}+l_{2} \leqslant 15 \mathrm{Mb} / \mathrm{s}$, the load transfer is established and one of the BSs is turned off. Fig. 2 also shows that BS 1 achieves its maximal utility when its load is slightly less than BS 2's load. The same result holds for BS 2 which is a symmetry on the diagonal line of Fig. 2. Due to space limitation, the figure corresponding to BS 2' utility is omitted.

Fig. 3 shows the expected utility of BS 1 when it knows its own load, but does not know BS 2's load. BS 1 calculates its expected utility based on the load distribution of BS 2. Blue line (with $\circ$ ) shows the results without cooperation and green line (with $\Delta$ ) shows the results with cooperation. Note that the expected utility goes down linearly with a constant gap from the non-cooperation case first, and then drops down to the non-cooperation case. This means that in the range with low load, the utility gain from cooperating is a constant that does not depend on the load. However, beyond $5 \mathrm{Mb} / \mathrm{s}$ it will be constrained by the BS's maximal capability $l^{\max }$. Due to symmetry, the same result holds for BS 2. This shows that both of the BSs would like to cooperate except when one knows itself has a 0 load. When a BS has no load, interim participation constraint is unsatisfied.

If BSs are asked whether to join the game before knowing their own loads, both of them have incentive to participate, which meets the ex ante participation constraint. Because before BS sharing, both BSs have expected utility of -125 , and after BS sharing both have expected utility of -100.2687 , which achieves $15.76 \%$ saving.

The simulation results also verify that the mechanism we design is incentive compatible. Fig. 4 shows that for BS 1, truth telling can achieve its maximal utility.

\section{B. BS 2 is lightly loaded}

In this case, the load of BS 1 is uniformly distributed in $[0,10 \mathrm{Mb} / \mathrm{s}]$ and the load of BS 2 is uniformly distributed in $[0,5 \mathrm{Mb} / \mathrm{s}]$.

Fig. 5(a) and Fig. 5(b) show the utility of BSs given a sample of their load combination $\left(l_{1}, l_{2}\right)$. Note that the two 


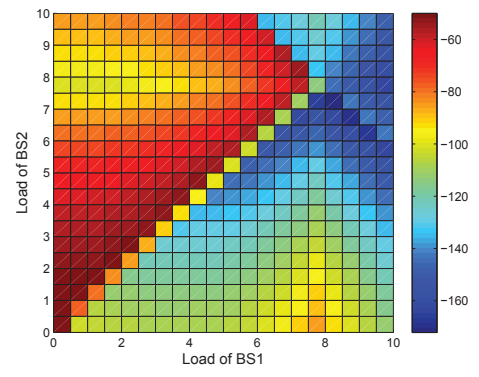

Fig. 2. Case A: The utility of BS 1 given the loads of both BSs.

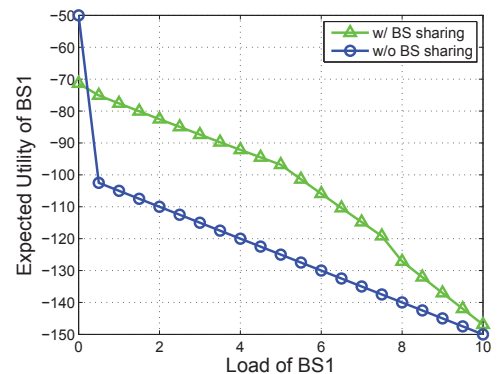

Fig. 3. Case A: The expected utility of BS 1 given the load of BS 1 .

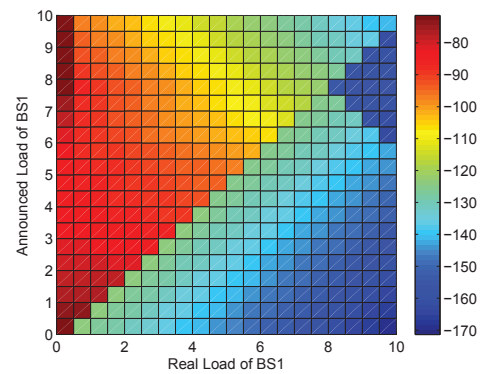

Fig. 4. Case A: The utility of BS 1 given its real load and its announced load.

utility functions are no longer symmetric now. BS 1 gets much higher utility gain than BS 2. The reason is that both of them are sure that BS 2 will be turned off in most cases. In order for BS 1 to tell its true load, large amount of money is transferred.

When each BS only knows its own load, the expected utility with cooperation and without cooperation are shown in Fig. 6(a) and Fig. 6(b), respectively. In this case, BS 2 with BS sharing has a worse expected utility whatever load it has. This causes BS 2 to have no incentive to cooperate, which contradicts the interim participation constraint.

\section{BS 2 is heavily loaded}

In this case, the load of BS 1 is uniformly distributed in $[0,10 \mathrm{Mb} / \mathrm{s}]$ and the load of BS 2 is uniformly distributed in $[5 \mathrm{Mb} / \mathrm{s}, 10 \mathrm{Mb} / \mathrm{s}]$.

When each BS only knows its own load, the expected utility with cooperation and without cooperation are shown in Fig. 7(a) and Fig. 7(b), respectively. In this case, BS 1 is indifferent

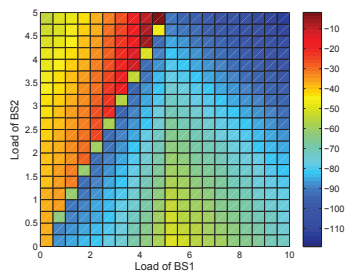

(a) The utility of BS 1 .

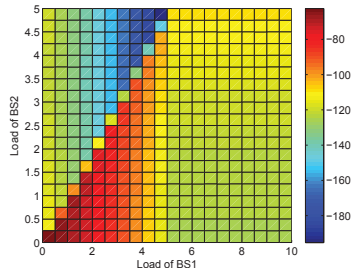

(b) The utility of BS 2 .
Fig. 5. Case B: The utility of BSs given their loads.

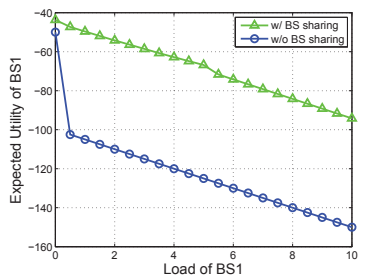

(a) The expected utility of BS 1 .

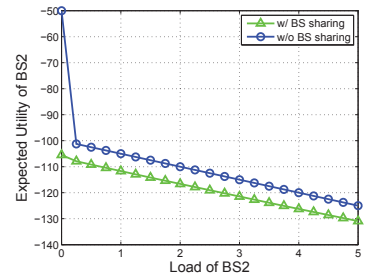

(b) The expected utility of BS 2 .
Fig. 6. Case B: The expected utility when each BS knows its own load.

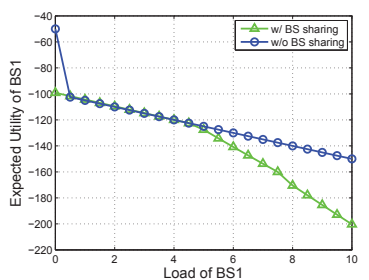

(a) The expected utility of BS 1 .

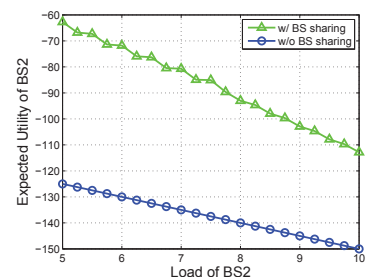

(b) The expected utility of BS 2 .
Fig. 7. Case C: The expected utility when each BS knows its own load.

to cooperate when its load is less than $5 \mathrm{Mb} / \mathrm{s}$. When its load is higher than $5 \mathrm{Mb} / \mathrm{s}$, BS 1 would not like to cooperate. For BS 2, it always has a positive utility gain from cooperating, even though the gain shrinks as its load grows.

\section{Both BSs are heavily loaded}

In this case, both BSs have load uniformly distributed in $[5 \mathrm{Mb} / \mathrm{s}, 10 \mathrm{Mb} / \mathrm{s}]$. As shown in Fig. 8, BS 1 has incentive to cooperate when its load is lower than $8.5 \mathrm{Mb} / \mathrm{s}$. The same result holds for BS 2 .

\section{E. Both BSs are lightly loaded}

In this case, both BSs have load uniformly distributed in $[0 \mathrm{Mb} / \mathrm{s}, 5 \mathrm{Mb} / \mathrm{s}]$. As shown in Fig. 9, the gap between BS 1's expected utility with BS sharing and without BS sharing is a constant and does not depend on BS 1's load. The reason is that in this case, one of the BSs can always be turned off and the load can always be transferred between BSs. The same result holds for BS 2 .

\section{F. Summary}

The expected utilities of BSs and the total energy saving in the five cases are summarized in Table I. They are the long 


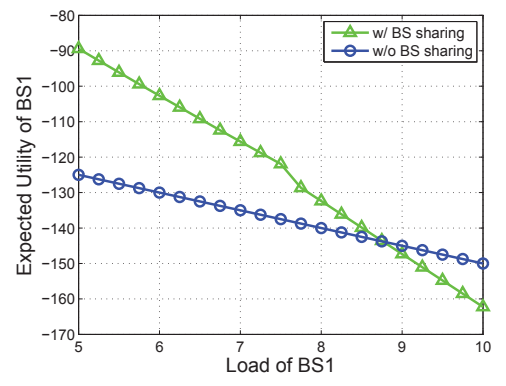

Fig. 8. Case D: The expected utility of BS 1 given the load of BS 1 .

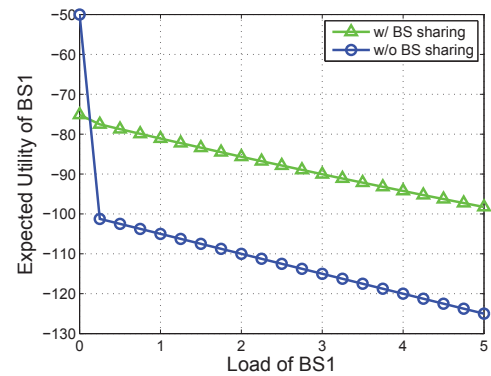

Fig. 9. Case E: The expected utility of BS 1 given the load of BS 1 .

term average utilities and also the expected utilities if they do not know their own load (which means before sampling). If their load distributions are similar, both of them would like to cooperate to achieve energy saving, as shown in the Case A, D and E. However, if one of them has a very different distribution compared with the other's, probably they cannot be both incentivized to cooperate, as shown in the Case $\mathbf{B}$ and C.

TABLE I

UTILITY SUMMARY IN THE FIVE CASES

\begin{tabular}{cccccc}
\hline \hline & Case A & Case B & Case C & Case D & Case E \\
\hline BS 1 w/o sharing & -125 & -125 & -125 & -137.5 & -112.5 \\
BS 1 w sharing & -103.29 & -68.88 & -138.06 & -124.71 & -87.60 \\
BS 2 w/o sharing & -125 & -112.5 & -137.5 & -137.5 & -112.5 \\
BS 2 w sharing & -103.29 & -118.91 & -87.44 & -124.71 & -87.60 \\
\hline $\begin{array}{c}\text { Total Energy } \\
\text { Saving }\end{array}$ & $15.76 \%$ & $19.31 \%$ & $13.31 \%$ & $9.30 \%$ & $20.44 \%$ \\
\hline
\end{tabular}

Another interesting finding is that the BS who has relatively higher traffic load is more likely to have utility gain through cooperating.

\section{CONCLUSION AND FUTURE WORK}

In this work, we show the potential of energy saving through operator cooperation even under the assumption that operators are self-interested and aim at maximizing their own utility. The mechanism designed in the work can easily be applied when the operators have similar traffic load distributions. However when the operators have very different traffic distributions, a subset of them may not have incentive to cooperate. Our work also shows that inter-operator BS sharing can save nearly $20 \%$ energy under the two operator case. Future work can include dynamic mechanism design where the traffic is correlated in the temporal domain, e.g., a Markov chain can be adopted to model the traffic load. How to motivate BSs with very different traffic distributions to cooperate will also be addressed in future work.

\section{ACKNOWLEDGMENT}

The authors would like to thank Professor Burkhard C. Schipper and Professor Xin Liu for their helpful discussions and continuous encouragement. This work is sponsored in part by the National Science Foundation of China (NSFC) under grant No. 61201191, the National Basic Research Program of China (973 Program: 2012CB316001).

\section{REFERENCES}

1] C.-L. I, "Green evolution of mobile communications (cmcc perspective)," 2012. [Online]. Available: http://www.greentouch.org/ uploads/documents

[2] M. A. Marsan, L. Chiaraviglio, D. Ciullo, and M. Meo, "Optimal energy savings in cellular access networks," in Communications Workshops, 2009. ICC Workshops 2009. IEEE International Conference on. IEEE, 2009, pp. 1-5.

[3] G. Auer, V. Giannini, C. Desset, I. Godor, P. Skillermark, M. Olsson, M. A. Imran, D. Sabella, M. J. Gonzalez, O. Blume et al., "How much energy is needed to run a wireless network?" Wireless Communications, IEEE, vol. 18, no. 5, pp. 40-49, 2011.

[4] E. Oh, B. Krishnamachari, X. Liu, and Z. Niu, "Toward dynamic energyefficient operation of cellular network infrastructure," Communications Magazine, IEEE, vol. 49, no. 6, pp. 56-61, 2011.

[5] Z. Niu, "Tango: traffic-aware network planning and green operation," Wireless Communications, IEEE, vol. 18, no. 5, pp. 25-29, 2011.

[6] S. Zhou, J. Gong, Z. Yang, Z. Niu, and P. Yang, "Green mobile access network with dynamic base station energy saving," in ACM MobiCom, vol. 9, no. 262, 2009, pp. 10-12.

[7] B. Leng, P. Mansourifard, and B. Krishnamachari, "Microeconomic analysis of base-station sharing in green cellular networks," in INFOCOM, 2014 Proceedings IEEE. IEEE, 2014, pp. 1132-1140.

[8] R. Berry, M. Honig, T. Nguyen, V. Subramanian, H. Zhou, and R. Vohra "On the nature of revenue-sharing contracts to incentivize spectrumsharing," in INFOCOM, 2013 Proceedings IEEE. IEEE, 2013, pp. $845-853$.

[9] W. Vickrey, "Counterspeculation, auctions, and competitive sealed tenders," The Journal of Finance, vol. 16, no. 1, pp. 8-37, 1961.

[10] E. H. Clarke, "Multipart pricing of public goods," Public choice, vol. 11, no. 1 , pp. $17-33,1971$

[11] T. Groves, "Incentives in teams," Econometrica: Journal of the Econometric Society, pp. 617-631, 1973.

[12] K. Arrow, The property rights doctrine and demand revelation under incomplete information. Economics and human welfare. New York Academic Press, 1979.

[13] C. d'Aspremont and L.-A. Gérard-Varet, "Incentives and incomplete information," Journal of Public Economics, vol. 11, no. 1, pp. 25-45, 1979

[14] C. Peng, S.-B. Lee, S. Lu, H. Luo, and H. Li, "Traffic-driven power saving in operational $3 \mathrm{~g}$ cellular networks," in Proceedings of the 17th annual international conference on Mobile computing and networking. ACM, 2011, pp. 121-132.

[15] J. Liu, T. Zhao, S. Zhou, Y. Cheng, and Z. Niu, "Concert: a cloud-based architecture for next-generation cellular systems," Wireless Communications, IEEE, vol. 21, no. 6, pp. 14-22, 2014.

[16] Wikipedia, "Bin packing problem," 2014, [Online; accessed 23-Sep2014]. [Online]. Available: http://en.wikipedia.org/wiki/Bin_packing_ problem

[17] R. B. Myerson and M. A. Satterthwaite, "Efficient mechanisms for bilateral trading," Journal of Economic Theory, vol. 29, no. 2, pp. 265$281,1983$. 